\title{
Tumor-associated macrophages as major source of APRIL in gastric MALT lymphoma
}

\author{
${ }^{*}$ Fabio Munari, ${ }^{1,2}$ *Silvia Lonardi, ${ }^{3}$ Marco A. Cassatella, ${ }^{4}$ Claudio Doglioni, ${ }^{5}$ Maria Giulia Cangi, ${ }^{5}$ Amedeo Amedei, 6,7 \\ Fabio Facchetti, ${ }^{3}$ Yoshinobu Eishi, ${ }^{8}$ Massimo Rugge, ${ }^{9}$ Matteo Fassan, ${ }^{9}$ Marina de Bernard, $, 1,10$ Mario M. D’Elios, ${ }^{6,7}$ and \\ William Vermi ${ }^{3}$
}

\begin{abstract}
${ }^{1}$ Venetian Institute of Molecular Medicine, Padova, Italy; ${ }^{2}$ Department of Biomedical Sciences, University of Padova, Padova, Italy; ${ }^{3}$ Department of Pathology, University of Brescia, Brescia, Italy; ${ }^{4}$ Department of Pathology and Diagnostics, Section of General Pathology, University of Verona, Verona, Italy; ${ }^{5}$ Department of Pathology, San Raffaele Scientific Institute, Milano, Italy; ${ }^{6}$ Department of Internal Medicine, University of Firenze, Firenze, Italy; ${ }^{7}$ Department of Biomedicine, Policlinico Careggi, Firenze, Italy; ${ }^{8}$ Department of Human Pathology, Graduate School of Medical Science, Tokyo Medical and Dental University, Tokyo, Japan; and ${ }^{~}$ Department of Medical-Diagnostic Sciences and Special Therapies, Surgical Pathology and Cytopathology Unit, and ${ }^{10}$ Department of Biology, University of Padova, Padova, Italy
\end{abstract}

\begin{abstract}
Lymphoid hyperplasia of gastric mucosa associated with Helicobacter pylori (HP) infection represents a preneoplastic condition of the mucosa associated lymphoid tissue (MALT), which may evolve to a B-cell lymphoma. While it is well established that the initial neoplastic proliferation of $B$ cells is antigen-driven and dependent on the helper activity of HP-specific $T$ cells, it needs to be elucidated which
\end{abstract}

cytokine or soluble factor(s) promote Bcell activation and lymphomagenesis. Herein, we originally report that gastric MALT Iymphoma express high levels of a proliferation inducing ligand (APRIL), a novel cytokine crucial in sustaining $B$-cell proliferation. By immunohistochemistry, we demonstrate that APRIL is produced almost exclusively by gastric lymphomainfiltrating macrophages located in close proximity to neoplastic B cells. We also show that macrophages produce APRIL on direct stimulation with both $H P$ and $H P$-specific T cells. Collectively, our results represent the first evidence for an involvement of APRIL in gastric MALT lymphoma development in $H P$-infected patients. (Blood. 2011;117(24):6612-6616)

\section{Introduction}

Helicobacter pylori $(H P)$ colonizes the human gastric mucosa and triggers a strong local inflammatory response. ${ }^{1}$ Chronic inflammation because of the persistence of $H P$ infection can give rise to organized lymphoid tissue in the gastric mucosa, the so-called mucosa-associated lymphoid tissue (MALT) that, in a small subset of individuals, can ultimately progress to low-grade gastric B-cell lymphoma of MALT type. $^{2}$ The current model of MALT lymphoma genesis assumes that one or more neoplastic clones, displaying characteristics of marginal zone B cells, originate from the organized MALT, colonize and replace the original follicles and eventually destroy the gastric glands to form lympho-epithelial lesions. ${ }^{3}$ Although MALT lymphoma usually grows slowly and has a low propensity to spread, a small percentage of cases undergo high-grade transformation. It is generally accepted that, in early stages of gastric lymphoma development, neoplastic growth is both antigen-driven and dependent of the helper activity of T cells specific for $H P^{2,4}$ The fact that the eradication of the bacterium leads to regression of the lymphoma, especially in its early stages, is consistent with such a postulate. ${ }^{5,6}$ However, less is known about the role of the host immune response in MALT lymphoma pathogenesis.

A proliferation inducing ligand $(A P R I L)$ is one of the most recently cloned members of the tumor necrosis factor $(T N F)$ family ${ }^{7-10}$ expressed by a variety of immune cells including neutrophils, monocytes, macrophages, dendritic cells, and T cells, but also epithelial cells and tumor cells. By binding to its BCMA and TACI receptors, APRIL promotes survival and proliferation of $\mathrm{B}$ cells as well as their differentiation to plasma cells. As recently reported, APRIL-transgenic mice develops lymphoid malignancies originating from peritoneal B-1 B cells. ${ }^{11}$ In addition, not only APRIL is expressed in a wide array of B-cell malignancies, including Hodgkin lymphomas, ${ }^{12}$ but high levels of APRIL in the serum of these patients correlate with poor prognosis. ${ }^{13}$ Consistently, in vitro experiments have clearly shown that APRIL promotes cell survival and proliferation of neoplastic B-cells (reviewed by Planelles et $\mathrm{al}^{13}$ and Roosnek et $\mathrm{al}^{14}$ ). Interestingly, APRIL expression in human non-Hodgkin B-cell lymphomas is found in neoplastic cells as well as in associated inflammatory cells, predominantly neutrophils. ${ }^{15}$

Herein, we report that gastric MALT lymphomas are particularly enriched in APRIL-expressing macrophages. We also show that, in vitro, $H P$ triggers an abundant release of APRIL by human monocyte-derived macrophages and that $H P$-specific T cells contribute to create an APRIL-enriched environment in MALT lymphoma. Collectively, our results provide the first evidence that APRIL may be involved in MALT lymphoma development in $H P$-infected patients.

\section{Methods}

\section{Tissues}

Tissues were obtained from the archive of the Department of Pathology (Spedali Civili di Brescia, Brescia, Italy, San Raffaele Hospital, Milan, Italy
Submitted June 25, 2010; accepted April 16, 2011. Prepublished online as Blood First Edition paper, April 29, 2011; DOI blood-2010-06-293266.
The publication costs of this article were defrayed in part by page charge payment. Therefore, and solely to indicate this fact, this article is hereby marked "advertisement" in accordance with 18 USC section 1734.

(C) 2011 by The American Society of Hematology 
and Padova Hospital). Samples included normal gastric mucosa (5 cases), $H P$-associated gastric lymphoid infiltrates Wotherspoon-Isaacson (WI) ${ }^{6}$ grades 1 ( 5 cases), 3 ( 3 cases), 4 ( 2 cases), and 5 ( 9 cases, resection specimens), gastric diffuse large B-cell lymphomas ( 5 cases) and $H P$ negative gastritis ( 6 cases). In the latter group $H P$ was assessed by histology (modified Giemsa staining) and confirmed by clinical history, rapid urease testing, and/or ELISA (Hp-specific IgG antibodies; Gastropanel, Biohit). A second group of samples consisted of matched endoscopic biopsies (preand posttreatment) from 6 patients with gastric MALT lymphoma who underwent clinical and histologic remission after $H P$-eradication therapy. WI-5 cases lack AP12/MALT1 translocation as proved by FISH analysis (MALT1 FISH deoxyribonucleic acid Probe; Split Signal; Dako Cytomation) and the large majority of them (7 of 9; 78\%) show $\operatorname{IgH}$ clonal rearrangements. For the IgH clonality assay, genomic deoxyribonucleic acid was extracted from tumor tissue sections using the QIAamp Tissue Kit (QIAGEN). PCR was performed using genomic deoxyribonucleic acid (200 ng) with primers targeting the variable region FR2 or FR3 and joining regions of the $\operatorname{IgH}$ gene. The amplified products were visualized in $10 \%$ polyacrylamide gel.

\section{Immunohistochemistry}

Four micron formalin-fixed paraffin embedded tissue sections were stained with anti-APRIL (Ms IgG1, Clone Aprily-2, Alexis Biochemicals) and anti-HP TMDU (Ms, Clone W4-1; provided by Prof Eishi, Department of Human Pathology, Graduate School of Medical Science, Tokyo Medical and Dental University, Tokyo, Japan). ${ }^{16}$ On appropriate antigen retrieval (water bath at $98^{\circ} \mathrm{C}$ for 40 minutes in ethylenediaminetetraacetic acid buffer $\mathrm{pH}$ 8.0), reactivity was revealed using NovoLink Polymer horseradish peroxidase linked (Novocastra Laboratories) followed by Diaminobenzidine (DAB). Characterization of APRIL positive cells was performed by double immunohistochemistry. After completing the first immune reaction, the second was realized using primary antibodies to the following antigens: CD11c (Clone 5D11; Novocastra Laboratories Ltd); CD68 (Clone KP-1; Dako); CD20 (Clone L26; Dako); CD163 (Clone 10D6; Thermo Scientific); CD1a (Clone 010; Dako); CD15 (Clone MMA; Thermo Scientific) and visualized using Mach 4-AP (Biocare Medical), followed by Ferangi Blue (Biocare Medical) as chromogen. Quantification of APRIL-expressing cells was performed on at least $5 \mathrm{HPF}$ (high power field) on sections double stained for APRIL and CD11c. Immunostained sections were photographed using the DP-70 Olympus digital camera mounted on the Olympus BX60 microscope, and the digital pictures (each corresponding to $0.036 \mathrm{~mm}^{2}$ ) were used for cell count. Values were expressed as the mean \pm SD.

\section{Bacteria and culture conditions}

$\mathrm{HP}$ strain 342 was maintained in $5 \% \mathrm{CO}_{2}$ at $37^{\circ} \mathrm{C}$ on Columbia agar plates supplemented with $5 \%$ horse blood. Colonies were inoculated into brain heart infusion broth containing $5 \%$ fetal bovine serum and were cultured for 2 days in rotary shaking at 180 revolutions per minute at $37^{\circ} \mathrm{C}$ under microaerophilic conditions.

\section{Macrophage differentiation}

Monocytes $\left(5 \times 10^{5}\right)$ isolated form buffy-coat and seeded in 24-well plates were cultured in RPMI 20\% FBS in the presence of macrophage colony stimulating factor (M-CSF; Immunologic Science; $100 \mathrm{ng} / \mathrm{mL}$ ) for a 6-day differentiation to M-CSF-differentiated human macrophages (MDM). MDM were cultured for 1 day in culture medium without M-CSF, before being exposed to bacteria $\left(5 \times 10^{5}\right.$ colony-forming unit $\left./ \mathrm{mL}\right)$.

\section{Real-time PCR analysis}

Total RNA was isolated from MDM using TRIzol solution (Invitrogen) according to the manufacturer's instructions. RNA was reverse-transcribed and amplified with the following primers: for GAPDH, 5'-AGCAACAGGGTGGTGGAC-3' and 5'-GTGTGGTGGGGGACTGAG-3'; for APRIL, 5'-AAGGGTATCCCTGGCAGAGT-3' and 5'-GCAGGACAGAGTGCTGCTT- $3^{\prime}$. After the amplification, data analysis was performed using the second derivative method algorithm. For each sample, the amount of messenger RNA (mRNA) of APRIL was expressed as the n-fold of the normalized amount of mRNA in untreated cells $(1$ arbitrary unit $=$ APRIL mRNA concentration/GAPDH mRNA concentration [both in fmoles/ $\mu \mathrm{L}]$ ).

\section{Generation of gastric T-cell clones and assay for T-cell clone helper function for APRIL production}

On approval of the local Ethical Committee (Department of Internal Medicine, University of Firenze), $5 \mathrm{HP}$-positive patients with gastric low-grade MALT lymphoma and 5 patients with uncomplicated $H P$-positive chronic gastritis gave their informed consent and were enrolled in the study. None of the patients had taken antibiotics or gastric proton pump inhibitors within 2 months before the study. Biopsy specimens were obtained during endoscopy from the gastric mucosa for (1) histology and $H P$ detection, (2) rapid urease test, and (3) culture of infiltrating lymphocytes. Diagnosis of $H P$ infection was based on positive urease test, histologic detection of $H P$, and positive 13C-urea breath test. Biopsy specimens of gastric mucosa were cultured for 7 days in complete medium supplemented with IL-2 $(50 \mathrm{U} / \mathrm{mL})$ to preferentially expand in vivo-activated T cells. Mucosal specimens were then disrupted, and single T-cell blasts were cloned by limiting dilution ( 0.3 cells/well) in round-bottom microwell plates containing $10^{5}$ irradiated PBMCs as feeder cells, phytohaemagglutinin $(0.5 \%$ $\mathrm{vol} / \mathrm{vol}$; Life Technologies) and IL-2 (20 U/mL). Cell surface marker analysis of T-cell clones was performed on a BD FACSCalibur, according to the CellQuest Version 5.1 software, using anti-CD3, anti-CD4, and anti-CD8 mAb purchased from $\mathrm{BD}$. T-cell clones were then screened in triplicate cultures for responsiveness to medium or $H P$ lysate $(10 \mu \mathrm{g} / \mathrm{mL})$ by measurement of $[3 \mathrm{H}]$ thymidine deoxyribose uptake after 60 hours in the presence of autologous PBMCs $\left(5 \times 10^{4}\right)$ as antigen presenting cells. Mitogenic index (ratio of mean counts per minute of stimulated to unstimulated cultures) $>5$ were considered positive. T-cell blasts of $H P$-specific clones $\left(8 \times 10^{5} / \mathrm{mL}\right)$ were cocultured for 16 hours with autologous macrophages $\left(4 \times 10^{5} / \mathrm{mL}\right)$ in the presence of medium or $H P$ antigen $(10 \mu \mathrm{g} / \mathrm{mL})$.

\section{APRIL protein detection in cells and in culture supernatant}

For the evaluation of the intracellular APRIL content, cells were collected and lysed in $20 \mathrm{mM}$ tris(hydroxymethyl)aminomethane, $150 \mathrm{mM} \mathrm{NaCl}$ $0.25 \% \mathrm{NP} 40$ at $4{ }^{\circ} \mathrm{C}$. Five micrograms of proteins were loaded on $4 \%-12 \%$ SDS-PAGE and transferred on nitrocellulose. APRIL was revealed using a specific mAb (Alexis Biochemical). The amount of extracellular APRIL was quantified by a commercial ELISA (Invitrogen), according to the manufacturer's instructions. For the evaluation of the intracellular APRIL content, MDM exposed to $H P$ were spread by cytospin on slides and stained for APRIL as indicated in "Immunohistochemistry."

\section{Statistical analysis}

Data are reported as the mean $\pm \mathrm{SD}$. The Student $t$ test and 1-way ANOVA were used for statistical analysis of the differences between experimental groups. $P$ values $\leq .05$ were considered significant.

\section{Results}

\section{HP-associated gastritis and gastric MALT lymphoma are} enriched in APRIL-containing tumor-associated macrophages

We initially evaluated by immunohistochemistry whether APRIL might be expressed in gastric MALT lymphomas. To this end, 9 cases of $\mathrm{HP}$-associated WI-5 cases lacking the API2/MALT1 translocation, as detected by FISH analysis (not shown), were used. Compared with normal gastric mucosa, in which APRIL reactivity is scanty (Figure 1A), we found numerous cells reacting to anti-APRIL in all WI-5 cases (Figure 1B). The large proportion of $\mathrm{APRIL}^{+}$cells showed a macrophage-like morphology and were admixed with $\mathrm{CD} 20^{+}$neoplastic B cells (Figure $1 \mathrm{~B}$ inset) and to surrounding plasma cells (not shown). By double immunohistochemistry of 5 representative cases, $\mathrm{APRIL}^{+}$cells were negative 


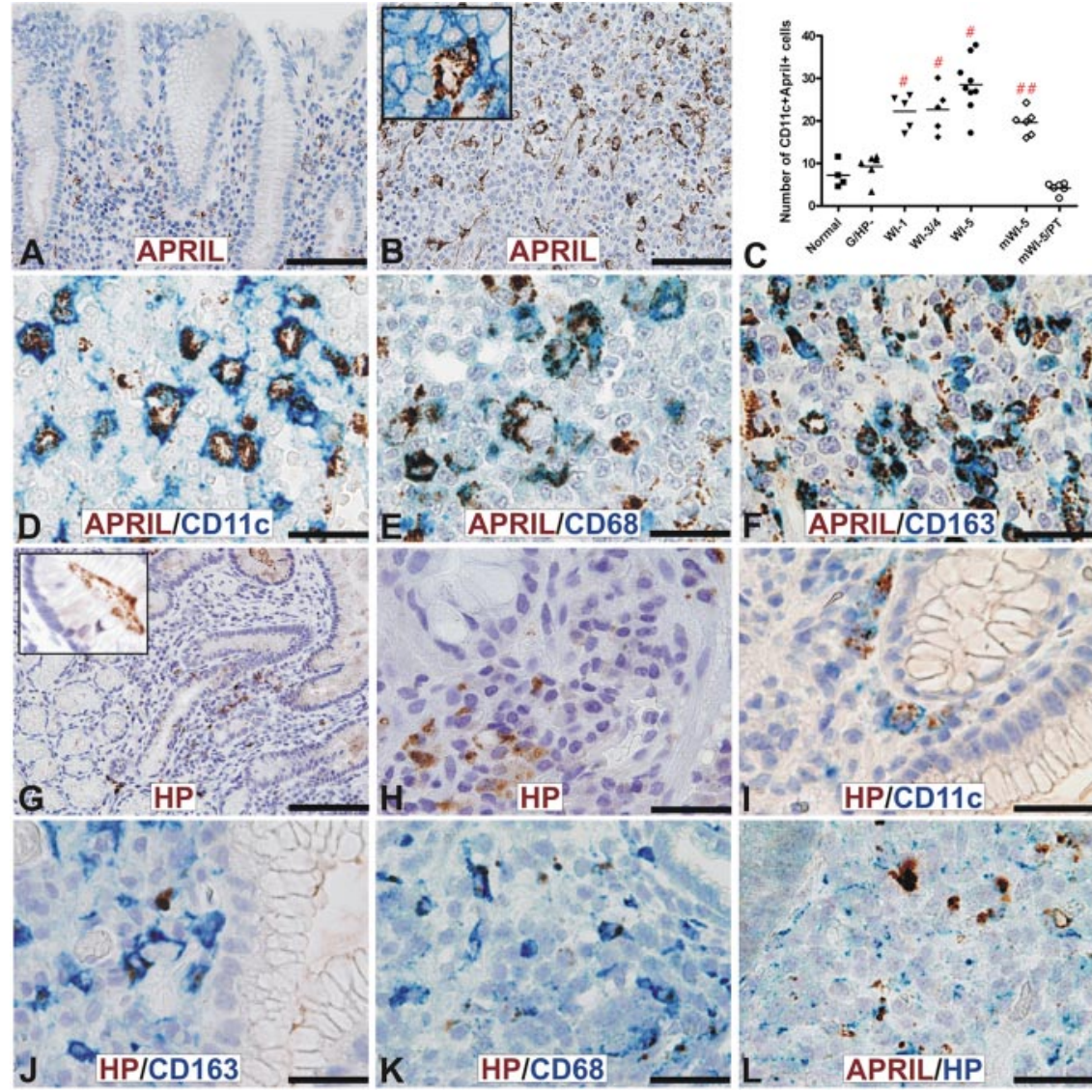

Figure 1. APRIL production by tissue macrophages. Sections are from normal gastric mucosa $(A), W I$ grade 5 (B,D-F) and grade 1 (G-L). Compared with normal gastric mucosa where APRIL reactivity is observed in rare large cells of the lamina propria (A), numerous APRIL ${ }^{+}$ cells are found $(B-C)$ surrounding $C D 20^{+}$neoplastic cells in MALT lymphoma (panel B inset) [red symbol in panel C indicates significance vs normal and $\mathrm{G} / \mathrm{HP}$ - $(\# P<.01)$ or vs $\mathrm{mWI}-5 / \mathrm{PT}(\# \# P<.01)]$. Double staining demonstrates that APRIL+ cells coexpress CD11c (D), CD68 (E), and CD163 (F). No APRIL reactivity is observed in neoplastic $\mathrm{B}$ cells (B,D-F). In WI grade 1 , the anti-HP antibody recognizes bacilli in the gastric mucous layer (panel $G$ inset); HP reactivity is also observed in the cytoplasm of numerous cells in the gastric lamina propria $(\mathrm{H})$. Double stain demonstrates that HP-containing cells are represented by macrophages coexpressing CD11C (I), CD163 $(\mathrm{J})$, and CD68 $(\mathrm{K})$ and some of them produce APRIL $(\mathrm{L})$. For immunohistochemistry, sections are counterstained with Meyer haematoxylin and secondary antibodies revealed with DAB (brown, anti-APRIL in panels A,B$\mathrm{D}, \mathrm{E}, \mathrm{F}, \mathrm{L} ;$ anti-HP in panels G-K), and Ferangi blue (blue, anti-CD11c in panels $\mathrm{D}, \mathrm{I}$; anti-CD68 in panels $\mathrm{E}, \mathrm{K}$; anti-CD163 in panels F,J; APRIL in panel L). Scale bar: $100 \mu \mathrm{m}$ (panels $A, B, G$ ) and $20 \mu \mathrm{m}$ (panels $\mathrm{D}-\mathrm{F}, \mathrm{I}-\mathrm{L}$ ). Legend in panel $\mathrm{C}$ : $\mathrm{G} / \mathrm{HP}$ - indicates $H P$-negative gastritis; mWI-5, matched WI-5; and mWI-5/PT, matched WI-5 posteradication treatment. for lymphoid markers CD20 and CD3 (Figure 1B inset and data not shown) but strongly reacting to CD11c, CD68 and CD163 but not to CD1a, thus confirming their macrophage identity (Figure 1D-F and data not shown). In addition to macrophages, a very small fraction of $\mathrm{CD}^{+} 5^{+}$polymorphonuclear cells also reacted to APRIL (not shown), while, in all cases, no reactivity for APRIL was observed in the large majority of neoplastic cells (Figure 1B-F). A further characterization of APRIL reactivity revealed numerous APRIL $^{+}$macrophages also in $H P$-associated WI grade 1 to 4 (Figure 1C). Of note, the number of APRIL-producing macrophages was significantly higher in WI lesions compared with normal gastric mucosa and $H P$-negative gastritis, being the mean number of $\mathrm{CD}_{11 \mathrm{c}^{+}} \mathrm{APRIL}^{+}$cells, respectively 22.2 [WI-1], 22.6 [WI-3/4], and 28.5 [WI-5] vs 7.2 [normal mucosa] and 9.2 [HP-negative gastritis] $(P<.01)$; on the contrary, differences among WI groups were not significant $(P=.07)$. Remarkably, in matched biopsies obtained from 6 MALT lymphoma patients, the number of $\mathrm{CD}_{11 \mathrm{c}^{+}} \mathrm{APRIL}^{+}$macrophages was dramatically reduced $(19.6$ vs $4.1 ; P<.01)$ in WI-5 cases, which underwent remission after $H P$-eradication treatment (Figure 1C). Notably, local production of APRIL was not exclusive of MALT lympho-

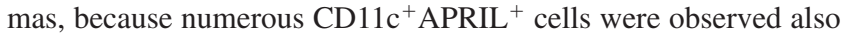
in cases of $H P$-negative gastric diffuse large B-cell lymphomas (not shown).

Together, these results strongly support the notion that macrophages represent a relevant source of APRIL available from the early phases of MALT lymphoma progression.

\section{HP-infected macrophages produce APRIL}

It was recently reported that cells containing $H P$-derived products can be found in the lamina propria of patients with gastric cancer and chronic gastritis, ${ }^{16}$ confirming early and more recent studies proving that mucosal invasion by $H P$ might occur in $H P$-dependent pathologies. ${ }^{17-20}$ Using the same antibody (recognizing an $\mathrm{HP}$ specific form of LPS), ${ }^{16}$ we were able to confirm this finding on cases of WI grade 1 densely colonized by $H P$. In particular, in addition to its classic localization in the gastric surface epithelium or in the spaces between mucous-secreting cells, we detected anti- $H P$ reactivity within the cytoplasm of macrophages localized in multiple foci in the gastric lamina propria (Figures 1G-H). Remarkably, $H P$-containing macrophages were positive for CD11c, CD163 and CD68 (Figure 1I-K), as well as intensely stained by the anti-APRIL antibody (Figure 1L). In parallel with these tissue sample evaluations, we subsequently addressed whether $H P$ could trigger, directly or indirectly, APRIL production by macrophages in vitro. APRIL, which is barely detectable in freshly purified monocytes, is actively produced at mRNA and protein level during monocytes differentiation to macrophages (Figure 2A-B); however, as quantified by ELISA of the culture supernatant (not shown), APRIL was not significantly released at any time point during the culture. HP exposure of MDM induces a time-dependent expression of APRIL mRNA (Figure 2C) in parallel with the extracellular accumulation of the related mature protein (Figure 2D). Addition of $H P$ to MDM also triggered a time-dependent accumulation of intracellular APRIL (Figure 2E).

\section{HP-specific T helper (Th) clones stimulate APRIL production from autologous macrophages}

Proliferation of neoplastic B cells in MALT lymphoma is sustained by both the antigen and the tumor-infiltrating helper T cells. ${ }^{21}$ To test the hypothesis that $H P$-specific $\mathrm{T}$ cells might sustain macrophage-derived APRIL production, we studied the helper function of 
A

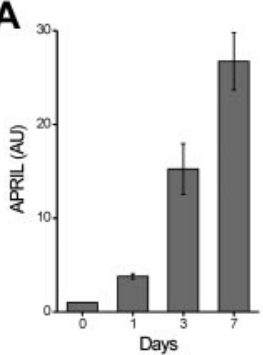

B
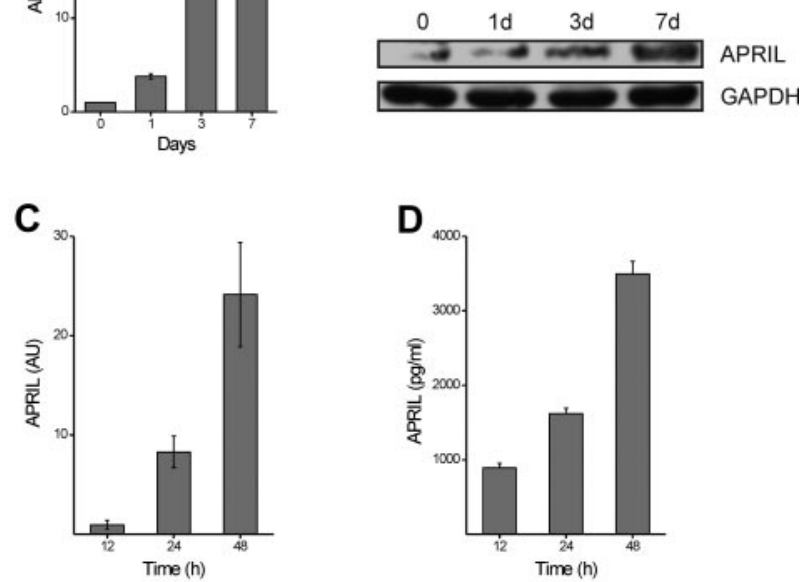

E

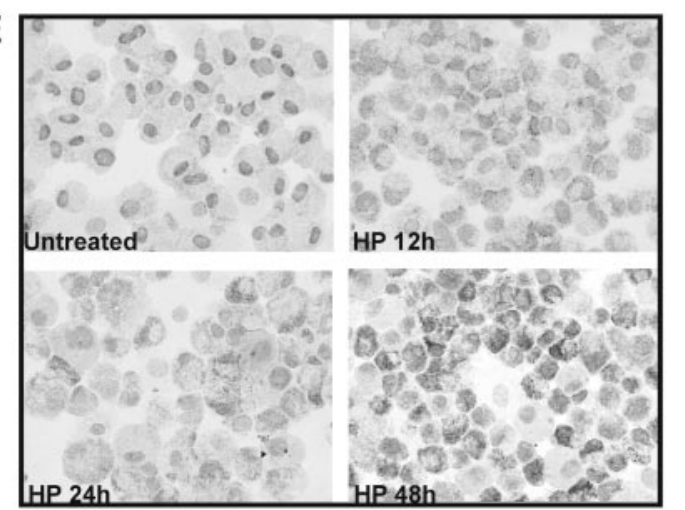

$\mathrm{F}$

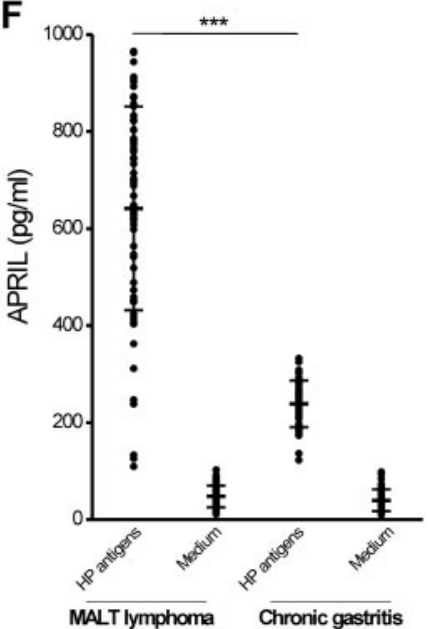

Figure 2. APRIL production during macrophage differentiation and exposure to $\boldsymbol{H P}$ or to $\boldsymbol{H P}$-specific T cells. Monocytes exposed to M-CSF were harvested the indicated time points and APRIL mRNA was quantified by real-time PCR (A); in parallel the APRIL protein cell content was determined by Western blot. GAPDH was used as marker of equal loading (B). M-CSF-differentiated macrophages (MDM) were exposed to $H P\left(5 \times 10^{5}\right.$ colony-forming unit $\left./ \mathrm{mL}\right)$. At the indicated time points cells were harvested for mRNA evaluation (C) and culture supernatant collected for APRIL content determination (D); AU indicates arbitrary units. In parallel, MDM cytospins were labeled for their intracellular APRIL content (E). HP-specific Th cells isolated from MALT lymphoma patients and from chronic gastritis patients were cocultured with autologous macrophages in the presence of medium or HP antigens and the APRIL production was assessed by ELISA (F). Results represent the APRIL levels induced by T-cell clones over the APRIL production in cultures of macrophages alone. ${ }^{\star * \star} P<.01$ clones of ex vivo freshly purified $\mathrm{T}$ cells obtained from antral biopsies of 5 patients with uncomplicated $H P$ chronic gastritis and $5 \mathrm{HP}$-associated gastric low-grade MALT lymphoma (see "Generation of gastric T-cell clones and assay for T-cell clone helper function for APRIL production" for details). By measuring the $[3 \mathrm{H}]$ thymidine deoxyribose uptake, we screened our T-cell clones for $H P$-induced proliferation in MHC-restricted conditions and obtained $71 \mathrm{HP}$-specific Th clones from patients with $H P$ associated low-grade MALT lymphoma and $50 \mathrm{HP}$-specific Th clones from patients with $H P$ chronic gastritis without MALT lymphoma. To test the helper ability of $H P$-specific $\mathrm{T}$ cells for APRIL production, $H P$-specific T-cell clones were cocultured for 16 hours with autologous monocyte-derived macrophages in the presence of $H P$ antigen. Remarkably, as demonstrated by ELISA, $H P$-specific Th clones from chronic gastritis and, even more strongly, those purified from MALT lymphoma cases, were able to stimulate the production of variable amounts of APRIL protein in an antigen-specific manner (Figure 2F).

\section{Discussion}

In this study, we have demonstrated that $H P$ infection in the gastric mucosa might sustain the local production of APRIL, a tumor necrosis factor superfamily member known to be important for B-cell development, maturation, and survival. In particular, numerous APRIL-producing macrophages are found in $H P$-infected chronic gastritis and mucosa-associated lymphoid tissue lymphoma biopsies, while they are very rare in normal mucosa. Notably, a fraction of APRIL-positive macrophages contain $\mathrm{HP}$ derived products; moreover, in vitro $H P$ infection induces expression of APRIL in monocyte-derived macrophages, thus indicating a primary role of the bacterium in promoting monocyte-derived cells to produce APRIL in infected gastric mucosa.

A large body of experimental and clinical evidence suggest that APRIL sustains B-cell transformation and progression of B-cell lymphomas. ${ }^{11-14}$ Different forms of B-cell lymphoma were found to contain APRIL-positive cells, that were represented either by neoplastic B-cells and by tumor associated immune cells, ${ }^{13}$ including macrophages. ${ }^{22}$ In keeping with these observations we have documented that $\mathrm{APRIL}^{+}$macrophages are also abundant in gastric diffuse large B-cell lymphomas devoid of HP infection. These observations indicate that the induction of APRIL in lymphomas partially reflect the local activation of tumor-associated macrophages likely mediated by different mechanisms. Craxton et al reported that human macrophages but not monocytes or monocytederived dendritic cells constitutively express high levels of APRIL. ${ }^{23}$ Furtermore, the expression of APRIL has been detected in macrophage-like cell lines, monocyte-derived macrophages and inflammatory macrophages. ${ }^{23-27}$ Stimuli mediating APRIL release by macrophages are largely unknown. We found that APRIL is gradually induced but not released by macrophages during M-CSFdependent differentiation from monocytes. However, we provide evidence that APRIL release by macrophages depends on $H P$, either after a direct stimulation by the bacteria, or by $H P$-specific $\mathrm{T}$ cells, especially those purified from overt MALT B-cell lymphoma.

The role of HP in gastric MALT lymphoma is well established, considering that chronic HP antigen stimulation is essential for their progression. Survival and proliferation of transformed B-cells in gastric MALT lymphoma require signals from tumor-associated T-cells or direct auto-antigen stimulation of neoplastic cells. 
(reviewed in Isaacson and $\mathrm{Du}^{3}$ and $\mathrm{D}^{\prime}$ Elios et $\mathrm{al}^{21}$ ). Our data suggest that tumor-associated macrophages might sustain progression of MALT lymphoma by releasing APRIL. We propose that $H P$-driven gastric inflammation leads to the generation of a local pool of macrophages that on $H P$ infection release large amount of APRIL. This functional loop can be further amplified and perpetuated by $H P$-specific T cells. Remarkably, as proof of principle of this postulate, APRIL-producing macrophages are dramatically reduced on lymphoma remission induced by $H P$ eradication.

Our results provide a new piece of information to $H P$ dependent MALT lymphomagenesis pointing to tumor-associated macrophages as contributors of tumor progression via APRIL release. Thus, based on these findings, we suggest that, among biologic therapies targeting $\mathrm{B}$ cells, ${ }^{28}$ blockade of APRIL might represent a novel additional therapeutic strategy for gastric MALT lymphoma, supporting the notion that the targeting tumorassociated macrophages and their products can be particularly relevant in lymphoid malignancies. ${ }^{29-30}$

\section{Acknowledgments}

The authors are grateful to Wilma Pellegrini, Cristina Rossini (supported by Fondazione Beretta, Brescia, Italy), Paola Bossini, and Laura Fappani for their technical support.

This work was supported by Progetto di Eccellenza Fondazione Cassa di Risparmio di Padova e Rovigo, Research Grant from the
University of Padova (CPDA074121/07), Associazione Italiana per la Ricerca sul Cancro Grant Regionale 2008, to M.d.B.; Fondazione Berlucchi, Brescia, Italy, to W.V., F.F., and S.L.; Ministero dell'Istruzione, dell'Università e della Ricerca (PRIN 2008C33HRL_003) and Ente Cassa di Risparmio di Firenze to M.M.D.; Ministero dell'Istruzione, dell'Università e della Ricerca (PRIN 2007H9AWXY), Fondazione Cariverona, and Associazione Italiana per la Ricerca sul Cancro (AIRC 5839), to M.A.C. S.L. is supported by "Borsa di studio Prof Roberto Tosoni" (Garda Vita, BCC del Garda).

\section{Authorship}

Contribution: W.V., M.M.D., M.A.C., and M.d.B. designed the study and wrote the manuscript; F.M., S.L., A.A., and M.C. performed research and analyzed data; and C.D., F.F., Y.E., M.R., and M.F. provided reagents, were involved in the interpretation of the results, read the manuscript, and gave comments.

Conflict-of-interest disclosure: The authors declare no competing financial interests.

Correspondence: William Vermi, MD, Department of Pathology, University of Brescia, Piazzale Spedali Civili, Brescia, Italy; e-mail: william.vermi@gmail.com; or Mario M. D’Elois, Department of Internal Medicine, University of Firenze, Firenze, Italy; e-mail: mariomilco.delios@unifi.it..

\section{References}

1. Marshall BJ. Helicobacter pylori. Am J Gastroen terol. 1994;89(8 suppl):S116-S128.

2. Hussell T, Isaacson PG, Crabtree JE, et al. The response of cells from low-grade B-cell gastric lymphomas of mucosa-associated lymphoid tissue to Helicobacter pylori. Lancet. 1993; 342(8871):571-574.

3. Isaacson PG, Du MQ. MALT Iymphoma: from morphology to molecules. Nat Rev Cancer. 2004; 4(8):644-653.

4. Farinha P, Gascoyne RD. Molecular pathogenesis of mucosa-associated lymphoid tissue lymphoma. J Clin Oncol. 2005;23(26):6370-6378.

5. Isaacson PG. Gastric MALT Iymphoma: from concept to cure. Ann Oncol. 1999;10(6):637-645.

6. Wotherspoon AC, Doglioni C, Diss TC, et al. Regression of primary low-grade B-cell gastric lymphoma of mucosa-associated lymphoid tissue type after eradication of Helicobacter pylori. Lancet. 1993;342(8871):575-577.

7. Hahne M, Kataoka T, Schroter M, et al. APRIL, a new ligand of the tumor necrosis factor family, stimulates tumor cell growth. J Exp Med. 1998; 188(6):1185-1190.

8. Kalled SL, Ambrose C, Hsu YM. The biochemistry and biology of BAFF, APRIL and their receptors. Curr Dir Autoimmun. 2005;8:206-242.

9. Kelly K, Manos E, Jensen G, et al. APRIL/ TRDL-1, a tumor necrosis factor-like ligand, stimulates cell death. Cancer Res. 2000;60(4): 1021-1027.

10. Lopez-Fraga M, Fernandez R, Albar JP, et al. Biologically active APRIL is secreted following intracellular processing in the Golgi apparatus by furin convertase. EMBO Rep. 2001;2(10):945-951.

11. Planelles L, Carvalho-Pinto CE, Hardenberg G, et al. APRIL promotes B-1 cell-associated neoplasm. Cancer Cell. 2004;6(4):399-408.

12. Burjanadze M, Matthes T, McKee T, et al. In situ detection of APRIL-rich niches for plasma-cell survival and their contribution to B-cell lymphoma development. Histol Histopathol. 2009;24(8): 1061-1066.

13. Planelles L, Medema JP, Hahne M, et al. The expanding role of APRIL in cancer and immunity. Curr Mol Med. 2008;8(8):829-844.

14. Roosnek E, Burjanadze M, Dietrich PY, et al. Tumors that look for their springtime in APRIL. Crit Rev Oncol Hematol. 2009;72(2):91-97.

15. Schwaller J, Schneider P, Mhawech-Fauceglia P, et al. Neutrophil-derived APRIL concentrated in tumor lesions by proteoglycans correlates with human B-cell lymphoma aggressiveness. Blood. 2007;109(1):331-338

16. Ito T, Kobayashi D, Uchida K, et al. Helicobacter pylori invades the gastric mucosa and translocates to the gastric lymph nodes. Lab Invest. 2008;88(6):664-681.

17. Andersen LP, Holck S. Possible evidence of invasiveness of Helicobacter (Campylobacter) pylori. Eur J Clin Microbiol Infect Dis. 1990;9(2):135138.

18. Ko GH, Kang SM, Kim YK, et al. Invasiveness of Helicobacter pylori into human gastric mucosa. Helicobacter. 1999;4(2):77-81.

19. Mai UE, Perez-Perez GI, Allen JB, et al. Surface proteins from Helicobacter pylori exhibit chemotactic activity for human leukocytes and are present in gastric mucosa. J Exp Med. 1992;175(2): 517-525.

20. Necchi V, Candusso ME, Tava F, et al. Intracellular, intercellular, and stromal invasion of gastric mucosa, preneoplastic lesions, and cancer by Helicobacter pylori. Gastroenterology. 2007; 132(3):1009-1023.

21. D'Elios MM, Amedei A, Del Prete G. Helicobacter pylori antigen-specific T-cell responses at gastric level in chronic gastritis, peptic ulcer, gastric cancer and low-grade mucosa-associated lymphoid tissue (MALT) lymphoma. Microbes Infect. 2003; 5(8):723-730

22. Chiu A, Xu W, He B, et al. Hodgkin lymphoma cells express $\mathrm{TACl}$ and $\mathrm{BCMA}$ receptors and generate survival and proliferation signals in response to BAFF and APRIL. Blood. 2007;109(2): 729-739.

23. Craxton A, Magaletti D, Ryan EJ, et al. Macrophageand dendritic cell-dependent regulation of human $B$-cell proliferation requires the TNF family ligand BAFF. Blood. 2003;101(11):4464-4471.

24. Lee SM, Jeon ST, Suk K, et al. Macrophages ex press membrane bound form of APRIL that can generate immunomodulatory signals. Immunology. 2010;131(3):350-356.

25. Sandberg WJ, Otterdal K, Gullestad L, et al. The tumour necrosis factor superfamily ligand APRIL (TNFSF13) is released upon platelet activation and expressed in atherosclerosis. Thromb Haemost,. 2009;102(4):704-710.

26. Gabay C, Krenn V, Bosshard C, et al. Synovial tissues concentrate secreted APRIL. Arthritis Res Ther. 2009;11(5):R144.

27. Belnoue E, Pihlgren M, McGaha TL, et al. APRIL is critical for plasmablast survival in the bone marrow and poorly expressed by early-life bone marrow stromal cells. Blood. 2008;111(5):27552764

28. Levesque MC. Translational Mini-Review Series on B Cell-Directed Therapies: Recent advances in B cell-directed biological therapies for autoimmune disorders. Clin Exp Immunol. 2009;157(2) 198-208.

29. Dave SS, Wright G, Tan B, et al. Prediction of survival in follicular lymphoma based on molecular features of tumor-infiltrating immune cells. N Engl J Med. 2004;351(21):2159-2169.

30. Steidl C, Lee T, Shah SP, et al. Tumor-associated macrophages and survival in classic Hodgkin's lymphoma. N Engl J Med. 2010;362(10):875-885. 


\section{Errata}

Johnson RM, Ravindranath Y, El-Alfy M, Goyette G Jr. Oxidant damage to erythrocyte membrane in glucose-6-phosphate dehydrogenase deficiency: correlation with in vivo reduced glutathione concentration and membrane protein oxidation. Blood. 1994;83(4): 1117-1123.

On page 1117 in the 15 February 1994 issue, there are errors in the third author's name (Mohsin El-Alfy). The correct name is Mohsen S. ElAlfy.

\section{Munari F, Lonardi S, Cassatella MA, et al. Tumor-associated macrophages as major source of APRIL in gastric MALT Iymphoma. Blood. 2011;117(24):6612-6616.}

On page 6616 in the 16 June 2011 issue, there is an error in the "Authorship." Under "Correspondence," the surname of the second corresponding author, shown as Mario M. D'Elois, is incorrect. The correct name is Mario M. D’Elios.

\section{Swierczek SI, Piterkova L, Jelinek J, et al. Methylation of $A R$ locus does not always reflect $\mathrm{X}$ chromosome inactivation state. Blood. 2012;119(13):e100-e109.}

On page e100 in the 29 March 2012 issue, the ninth author's middle initial was omitted. The correct name is Bradley R. Cairns. In the "Authorship" on page e109, the initials "B.C." in the "Contribution" should have read "B.R.C." The errors have been corrected in the online version, which now differs from the print version.

\section{Duhen T, Duhen R, Lanzavecchia A, Sallusto F, Campbell DJ. Functionally distinct subsets of human FOXP3 ${ }^{+}$Treg cells that phenotypically mirror effector Th cells. Blood. 2012;119(19):4430-4440.}

On pages 4430 and 4439 in the 10 May 2012 issue, there are errors in the byline and in Figure 7. The first and second authors (Thomas Duhen and Rebekka Duhen) are also affiliated with the Department of Immunology, University of Washington School of Medicine, Seattle, WA, which should have been denoted by their names in the byline with a superscripted 2 . As a result of this change, the affiliations for all authors will read as shown.

\section{Thomas Duhen, ${ }^{1,2}$ Rebekka Duhen, ${ }^{1,2}$ Antonio Lanzavecchia, ${ }^{3}$ Federica Sallusto, ${ }^{3}$ and Daniel J. Campbell ${ }^{1,2}$}

${ }^{1}$ Benaroya Research Institute, Seattle, WA; 2Department of Immunology, University of Washington School of Medicine, Seattle, WA; and ${ }^{3}$ Institute for Research in Biomedicine, Bellinzona, Switzerland

In Figure 7B, the bottom panels are mislabeled. Instead of reading "Th17"and "Th17-like," the labels should have read "Th1" and "Th1-like." The errors have been corrected in the online version, which now differs from the print version. 


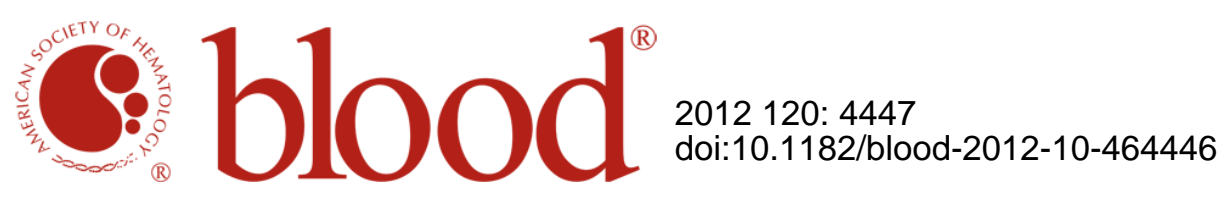

\section{Munari F, Lonardi S, Cassatella MA, et al. Tumor-associated macrophages as major source of APRIL in gastric MALT lymphoma. Blood. 2011;117(24):6612-6616.}

Updated information and services can be found at:

http://www.bloodjournal.org/content/120/22/4447.2.full.html

Articles on similar topics can be found in the following Blood collections

Information about reproducing this article in parts or in its entirety may be found online at:

http://www.bloodjournal.org/site/misc/rights.xhtml\#repub_requests

Information about ordering reprints may be found online at:

http://www.bloodjournal.org/site/misc/rights.xhtml\#reprints

Information about subscriptions and ASH membership may be found online at:

http://www.bloodjournal.org/site/subscriptions/index.xhtml

Blood (print ISSN 0006-4971, online ISSN 1528-0020), is published weekly by the American Society of Hematology, 2021 L St, NW, Suite 900, Washington DC 20036.

Copyright 2011 by The American Society of Hematology; all rights reserved. 